\title{
EL EMPRENDIMIENTO EN LOS ESTUDIANTES DE LA UNMSM Y SU RELACIÓN CON ALGUNAS VARIABLES SOCIODEMOGRÁFICAS
}

\section{THE ENTREPRENEURSHIP IN THE STUDENTS OF THE UNMSM AND ITS RELATION WITH SOME VARIABLES SOCIODEMOGRAFIC}

\author{
Alejandro Loli P. ${ }^{1}$, Javier Del Carpio G., Elsa La Jara G. \\ Universidad Nacional Mayor de San Marcos, Lima, Perú \\ (RECIBIDO EL 4/01/2009, ACEPTADO EL 07/04/2009)
}

\begin{abstract}
RESUMEN
El estudio tiene como objetivo conocer la actitud emprendedora de los estudiantes de la Universidad Nacional Mayor de San Marcos y su relación con algunas variables demográficas en una muestra de 182 sujetos que realizan estudios universitarios, en su mayoría de ingeniería en las diversas especialidades, encontrándose todos ellos entre el tercero y quinto año de estudios.
\end{abstract}

Los resultados nos indican que la mayoría de los estudiantes universitarios tienen una actitud positiva hacia la creatividad y el empredimiento; igualmente, existe una correlación significativa y positiva entre sus componentes. Sobre las variables demográficas y psicosociales y su relación con los componentes, existe diferencia significativa de acuerdo a los grupos de edad, ciclos de estudio cursado, Facultad y Escuela Académico Profesional de pertenencia, desarrollo de emprendimiento y sector de actividad para el emprendimiento; es decir, la intervención de dichas variables permiten inferir diferencias de percepción respecto a algunos factores de la creatividad y el empredimiento.

Palabras clave: Creatividad, emprendimiento, estudiantes universitarios, Perú.

\begin{abstract}
This study has as an objective to know the entrepreneurship attitude of Universidad Nacional Mayor de San Marcos students and their relationship to some demographic variables, which have been taken from a sample of 182 students belonging to different engineering specialties and from third to the fifth year of studies.

The results indicate that the majority of the students have a positive attitude towards the creativity and entrepreneurship, in the same way, there is a significative and positive correlation between their components. With respect the demographics and psychosocial variables, there is a difference significative according to the age groups, year of study, development of entrepreneurship, College and Academic Department, and kind of

1 Docente investigador del Instituto de Investigaciones Psicólogicas de la Facultad de Psicología, Universidad Nacional Mayor de San Marcos, Lima-Perú. E-mail: alolip@unmsm.edu.pe
\end{abstract}


entrepreneurship activity, it could say, the presence of those variables allow to infer that there are differences respect some creativity and entrepreneurship factors.

Keywords: Creativity, entrepreneurship, college students, Peru.

\section{INTRODUCCIÓN}

En las últimas décadas, el Perú vivió situaciones de crisis política, económica y social, comprometiendo incluso la ética y la moral de nuestra sociedad. Este hecho llevó a las poblaciones rurales de la selva, sierra y costa a migrar a las zonas urbanas en búsqueda de un mejor nivel de vida, a través de un empleo digno o de mayor seguridad para la preservación de su vida.

De este modo, el crecimiento demográfico de la población en las zonas urbanas superó todo cálculo, haciendo difícil el acceso a un empleo digno y una vida aceptable. La alternativa más viable que encontraron dichos migrantes fue el trabajo independiente, creando su propia fuente de trabajo, desde la informalidad inicial hasta la formación de organizaciones empresariales de importancia.

¿Cómo llamarle a este fenómeno que nace en medio de la carencia, la necesidad, la escasa oferta de oportunidades de empleo y el surgimiento de nuevas fuentes de trabajo? La única respuesta posible se llama emprendedorismo.

El emprendedor es aquel que detecta una oportunidad, la analiza, la estudia y se proyecta para crear una organización; es aquella persona que posee olfato y que sabe encontrar las oportunidades; es alguien que cree en sus ideas y que es capaz de llevarlas a cabo, de encontrar la manera de materializar sus sueños; alguien que aprende de sus errores, que no se inmoviliza o abandona cuando se equivoca o fracasa, que intenta salir adelante pese a las dificultades que encuentra (M. Rodríguez, 2007). El empredendor “....es un innovador, un gran estratega, (...) tiene personalidad creativa, siempre desafiando lo desconocido, transformando posibilidades en oportunidades" (Gerber, 1996, en J. Bóveda, 2004); “... emprededores pueden ser definidos como individuos que innovan, identifican y crean oportunidades de negocios ..." (Lezana y Tonelli, 1998, en J. Bóveda, 2004).

El estudio de N. Espinoza (2004), sobre aptitudes y actitudes empresariales de los estudiantes de administración en tres universidades públicas de Lima y Callao, Perú, en una muestra de 200 alumnos, encontró que el 56.5\% no tiene trabajo dependiente, y el $43.5 \%$ tiene trabajo dependiente. En el primer caso, el $16.81 \%$, indica tener negocio propio y $83.19 \%$, no tener negocio propio; en el segundo caso, el $9.5 \%$ señala tener negocio propio, y el resto no lo tiene.

Aquellos que no trabajan ni tienen negocio propio (47\%), tienen como objetivo concluir sus estudios $(27.65 \%)$, realizar sus prácticas profesionales $(26.59 \%)$, crear su negocio propio $(25.55 \%)$, buscar trabajo $(17.02 \%)$ y salir al extranjero $(3.19 \%)$. Datos con los que el autor trata de demostrar la escasa actitud de emprendimiento de los estudiantes, explicando que aquellos que dicen tener negocio propio se refieren al negocio de sus padres. No existen estrategias pedagógicas en las facultades de administración de las universidades públicas, no están orientadas a formar empresarios ni a promover aptitudes y actitudes empresariales. 
Otro estudio desarrollado con estudiantes universitarios es de F. A. Delicio (2007), con el objeto de conocer acerca de la existencia de una vocación emprendedora entre los estudiantes y las motivaciones que llevan a dicha vocación en la Facultad de Ciencias Económicas y Sociales, de la Universidad Nacional de Mar del Plata - UNMDP, Argentina. Se contó con una muestra 47 sujetos del último año de la carrera de contador público y administración. Los resultados nos indican que la proporción de estudiantes con vocación emprendedora es baja (14.89\%); el ejemplo familiar como modelo a seguir para desarrollar una vocación emprendedora entre los estudiantes es escasa; la experiencia laboral y la formación académica que reciben de la universidad es un buen complemento para intentar su propio emprendimiento, pero insuficiente; los alumnos demandan más cuestiones relacionadas con la posibilidad de desarrollar una actividad emprendedora. La universidad está preparando alumnos para la gran empresa, que no es precisamente la principal generadora de empleo.

Fernando Graña y Mariel Fornoni (2002) realizaron una investigación sobre la vocación emprendedora en una muestra de 189 sujetos de las Facultades de Ciencias Económicas y Sociales de dos universidades de Mar del Plata, Argentina, encontraron que el 35.5\% de los estudiantes posee vocación emprendedora; el $17.5 \%$ tiene comportamiento emprendedor, iniciando una empresa propia; el $8 \%$ tienen intención emprendedora, con un proyecto concreto para crear una nueva empresa, y el $10 \%$ tiene una actitud emprendedora, admitiendo la posibilidad de iniciar un nuevo negocio como opción de carrera o salida laboral.

El Centro de Desarrollo Emprendedor del Instituto de Estudios Empresariales de Montevideo (IEEM) de la Universidad de Montevideo (2007), en el marco del proyecto Global Enterpreneurship Monitor (GEM), publicó algunos resultados de una investigación realizada sobre las relaciones entre la actividad emprendedora y el crecimiento económico en estudiantes universitarios. Según el informe, 13 de cada 100 personas estaban desarrollando, a mediados de 2006, algún tipo de empresa naciente. Según el PBI per cápita, estas cifras colocan a Uruguay en el séptimo lugar dentro del ranking de actividad emprendedora para los "países pobres". Sin embargo, cuando a los jóvenes se les formula la siguiente pregunta: ¿Qué ha hecho usted en los últimos 12 meses para poder emprender su negocio?; la mayoría respondió nada. Situación muy común en jóvenes universitarios, cuya reacción es básicamente actitudinal, de optimismo, y donde la Universidad debe jugar un papel más activo para el fomento de la actitud emprendedora (M. Olivero, 2007).

Emilio Cevallos V. (2007) realizó un estudio acopiando información de diferentes países de América Latina sobre restricciones del entorno a la competitividad empresarial; en algunos casos, realiza entrevistas a directores de empresas, administradores, gerentes y propietarios, y encuentra que la mayoría de países están más preocupados por temas como la asociatividad empresarial (El Salvador, Colombia, Chile), cultura empresarial (Panamá), aspectos de índole social (Venezuela), que por emprendedorismo (Bolivia y Argentina). En el caso de Bolivia, el $83 \%$ estaba interesado en abrir su propio negocio, y en el caso Argentino, si bien había interés por el emprendedorismo, fue muy escaso; sus preocupaciones estaban orientadas hacia la venta directa de sus productos y la compra de sus materias primas e insumos en forma individual (94\%). 
Juan A. Moriano L. (2005), por otro lado, desarrolla una investigación con el objeto de explicar y predecir la intención de emprender, en una muestra de 2000 estudiantes, la mayor parte de ellos residentes en Castilla y León, España. Los resultados nos muestran que sólo el $13.5 \%$ de los participantes muestra mayor intención de desarrollar su carrera profesional a través del autoempleo que trabajando por cuenta ajena. Sin embargo, el estudio señala que sólo el $4.11 \%$ creará efectivamente su propia empresa. Se sigue pensando que la mejor opción laboral es conseguir un trabajo seguro para toda la vida.

En concordancia con estudiosos del tema, hay emprendedores que surgen espontáneamente como respuesta a las necesidades y las carencias, pero también aprendiendo de sus experiencias e interacciones sociales, y otros que nacen con algunas cualidades innatas que son fortalecidas durante su desarrollo. Se dice, igualmente, que todos los seres humanos somos emprendedores por naturaleza. Es posible que estos y otros factores influyan en la formación de los emprendedores; por ahora, queremos indagar lo que pasa entre nuestros estudiantes universitarios. Hay un discurso instituido, desde algún tiempo, que las universidades juegan un papel importante (M. Olivero, 2007) o tienen que jugar un papel trascendente en la formación de jóvenes emprendedores.

Por tanto, el objetivo de este estudio fue conocer la actitud emprendedora de los estudiantes de la Universidad Nacional Mayor de San Marcos y su relación con algunas variables demográficas y sociales.

\section{MÉTODO}

\section{Muestra}

La muestra estuvo constituida por 182 estudiantes de las Facultades de Ingeniería en sus diversas especialidades y de la Facultad de Psicología de la especialidad organizacional y social. De ellos, 55 (30.21\%) fueron de sexo femenino y 127 (69.78\%) de sexo masculino. La distribución de la muestra de acuerdo a la edad es la siguiente:

\begin{tabular}{ccccl}
\hline \multicolumn{5}{c}{ Edad } \\
\hline $15-20$ & $21-25$ & $26-30$ & 31 a más & Total \\
33 & 118 & 27 & 4 & $\mathbf{1 8 2}$ \\
\hline
\end{tabular}

\section{Instrumento}

El instrumento fue construido por Alejandro E. Loli Pineda (2008), exclusivamente para propósitos de la presente investigación. Dicho Inventario de Creatividad y Emprendimiento está conformado por 12 factores: creatividad e iniciativa, optimismo, perseverancia, compromiso con sus convicciones, confianza en sí mismo, propensión al riesgo, capacidad de adaptación, liderazgo, independencia y autonomía, necesidad de logro, visión del futuro y competencias; haciendo un total de 94 ítemes entre todos sus componentes, los cuales cuentan con seis alternativas de respuesta que van desde totalmente en desacuerdo (1) a totalmente de acuerdo (6). 
En concordancia con el coeficiente alpha de Cronbach, los diferentes factores o componentes cumplen con el criterio de estabilidad y confiabilidad, superando el mínimo (0.20) exigido para estos fines. La confiabilidad general lograda en el procesamiento de datos del presente estudio fue de 0.958; por tanto, altamente estable y confiable. En la siguiente tabla se puede observar el nivel de confiabilidad de cada componente.

Tabla de confiabilidad para cada uno de los componentes

\begin{tabular}{lc}
\hline \multicolumn{1}{c}{ Factores o componentes } & Confiabilidad \\
\hline Creatividad e iniciativa & 0.707752579 \\
Optimismo & 0.608352597 \\
Perseverancia & 0.700403572 \\
Compromiso con sus convicciones & 0.451153213 \\
Confianza en sí mismo & 0.741732155 \\
Propension al riesgo & 0.616841774 \\
Capacidad de adaptación & 0.610742857 \\
Iiderazgo & 0.787749399 \\
Independencia y autonomía & 0.535675102 \\
Necesidad de logro & 0.566236647 \\
Visión del futuro & 0.702530314 \\
Competencias & 0.776619019 \\
Confiabilidad total & 0.958 \\
\hline
\end{tabular}

Cronbach's Alpha

\section{Procesamiento de datos}

Se utilizó el paquete estadístico SPSS (Statistical Package for the Social Sciences) para el tratamiento estadístico. De manera especial se utilizó el estadístico de confiabilidad Alpha de Cronbatch para ver los niveles de confiabilidad de la prueba total y de cada uno de los factores que la componen, la correlación de Spearman para establecer el grado de significancia en la relación entre los componentes, y las pruebas no paramétricas de comparación para dos gupos de Mann-Withney y para más de dos grupos de Kruskal Wallis.

\section{RESULTADOS}

\section{Actitud de los estudiantes universitarios hacia el emprendimiento}

Una evaluación general de la Tabla N. ${ }^{\circ} 1$, sobre la creatividad y el emprendimiento de los estudiantes de la Universidad Nacional Mayor de San Marcos, Lima-Perú, a través de los 12 factores que conforman el Inventario, demuestra que tienen una actitud positiva, siendo el $71.4 \%$ de los encuestados los que se encuentran en un nivel alto, con tendencia a incrementarse a un nivel muy alto. 
Tabla N. ${ }^{0}$ 1. Actitud para el emprendimiento.

\begin{tabular}{clcc}
\hline & & Frecuencia & Porcentaje válido \\
\hline \multirow{4}{*}{ Válidos } & Medio & 12 & 6.6 \\
& Alto & 130 & 71.4 \\
& Muy alto & 40 & 22.0 \\
& Total & 182 & 100.0 \\
\hline
\end{tabular}

\section{Relación entre los factores y la creatividad y el emprendimiento}

En la Tabla N. ${ }^{\circ} 2$ se encuentra el análisis de correlación de los factores de la creatividad y el emprendimiento de los estudiantes de la Universidad Nacional Mayor de San Marcos. Nos muestra que existe una correlación significativa y positiva entre creatividad e iniciativa con optimismo $(\mathrm{r}=.58 * * *)$, perseverancia $(\mathrm{r}=.59 * * *)$, compromiso con sus convicciones $(\mathrm{r}=.49 * * *)$, confianza en sí mismo $\left(\mathrm{r}=.65^{* * *}\right)$, propensión al riesgo $(\mathrm{r}=.57 * * *)$, capacidad de adaptación $(\mathrm{r}=.54 * * *)$, Liderazgo $\left(\mathrm{r}=.65^{* * *}\right)$, independencia y autonomía $(\mathrm{r}=.50 * * *)$, necesidad de logro $(\mathrm{r}=.62 * * *)$, visión del futuro $(\mathrm{r}=.67 * *)$, competencias $(\mathrm{r}=.62 * * *)$; es decir, a mayor creatividad e iniciativa mayor optimismo, perseverancia, compromiso con sus convicciones, confianza en sí mismo, mayor propensión al riesgo, mayor capacidad de adaptación, liderazgo, independencia y autonomía, necesidad de logro, visión del futuro y mayores competencias.

Tabla $\mathbf{N}^{0}$ 2. Correlación entre creatividad e iniciativa y los demás factores del emprendimiento.

\begin{tabular}{lcc}
\hline & \multicolumn{2}{c}{ CREATIVIDAD E INICIATIVA } \\
\cline { 2 - 3 } & \multicolumn{1}{c}{ r } & p-value \\
\hline Creatividad e iniciativa & 1 & - \\
Optimismo & 0.58231524 & 0.000001 \\
Perseverancia & 0.5867387 & 0.000001 \\
Compromiso con sus convicciones & 0.49014013 & 0.000001 \\
Confianza en sí mismo & 0.64794111 & 0.000001 \\
Propensión al riesgo & 0.56800749 & 0.000001 \\
Capacidad de adaptación & 0.53570237 & 0.000001 \\
Liderazgo & 0.64525514 & 0.000001 \\
Independencia y autonomía & 0.50160374 & 0.000001 \\
Necesidad de logro & 0.61715082 & 0.000001 \\
Visión del futuro & 0.6703369 & 0.000001 \\
Competencias & 0.6225503 & 0.000001 \\
\hline
\end{tabular}


La Tabla N. ${ }^{\circ} 3$ nos muestra que existe una correlación significativa y positiva entre perseverancia y creatividad e iniciativa $(\mathrm{r}=.59 * * *)$, optimismo $\left(\mathrm{r}=.65^{* * *}\right)$, compromiso con sus convicciones $(\mathrm{r}=.52 * * *)$, confianza en sí mismo $(\mathrm{r}=.74 * * *)$, propensión al riesgo $(\mathrm{r}=.58 * * *)$, capacidad de adaptación $(\mathrm{r}=.64 * * *)$, Liderazgo $(\mathrm{r}=.54 * * *)$, independencia y autonomía $(\mathrm{r}=.37 * * *)$, necesidad de logro $\left(\mathrm{r}=.41^{* * *}\right)$, visión del futuro $(\mathrm{r}=.58 * * *)$, competencias $(\mathrm{r}=.59 * * *)$; es decir, a mayor perseverancia mayor creatividad e iniciativa, mayor optimismo, compromiso con sus convicciones, confianza en sí mismo, mayor propensión al riesgo, mayor capacidad de adaptación, liderazgo, independencia y autonomía, necesidad de logro, visión del futuro y mayores competencias.

Tabla N. ${ }^{\circ}$. Correlación entre perseverancia y los demás factores del emprendimiento.

\begin{tabular}{lcc}
\hline & \multicolumn{2}{c}{ PERSEVERANCIA } \\
\cline { 2 - 3 } & $\mathbf{r}$ & p-value \\
\hline Creatividad e iniciativa & 0.5867387 & 0.000001 \\
Optimismo & 0.65497568 & 0.000001 \\
Perseverancia & 1 & $\cdot$ \\
Compromiso con sus convicciones & 0.52051532 & 0.000001 \\
Confianza en sí mismo & 0.73731948 & 0.000001 \\
Propensión al riesgo & 0.58466438 & 0.000001 \\
Capacidad de adaptación & 0.64833287 & 0.000001 \\
Liderazgo & 0.54295948 & 0.000001 \\
Independencia y autonomía & 0.37948882 & 0.000001 \\
Necesidad de logro & 0.41359165 & 0.000001 \\
Visión del futuro & 0.58151162 & 0.000001 \\
Competencias & 0.59059288 & 0.000001 \\
\hline
\end{tabular}

La Tabla N. ${ }^{\circ} 4$ nos muestra que existe una correlación significativa y positiva entre compromiso con sus convicciones y creatividad e iniciativa $(\mathrm{r}=.49 * * *)$, optimismo $\left(\mathrm{r}=.48^{* * *}\right)$ perseverancia $(\mathrm{r}=.52 * * *)$, confianza en sí mismo $(\mathrm{r}=.53 * * *)$, propensión al riesgo $(\mathrm{r}=.52 * * *)$, capacidad de adaptación $(\mathrm{r}=.60 * * *)$, Liderazgo $(\mathrm{r}=.48 * * *)$, independencia y autonomía $\left(\mathrm{r}=.27^{* * *}\right)$, necesidad de logro $\left(\mathrm{r}=.35^{* * *}\right)$, visión del futuro $\left(\mathrm{r}=.44^{* * *}\right)$, competencias $\left(\mathrm{r}=.46^{* * *}\right)$; es decir, a mayor compromiso con sus convicciones mayor creatividad e iniciativa, optimismo, perseverancia, compromiso con sus convicciones, confianza en sí mismo, mayor propensión al riesgo, mayor capacidad de adaptación, liderazgo, independencia y autonomía, necesidad de logro, visión del futuro y mayores competencias.

La Tabla N. ${ }^{\circ} 5$ nos muestra que existe una correlación significativa y positiva entre optimismo y creatividad e iniciativa $\left(\mathrm{r}=.58^{* * *}\right)$, perseverancia $\left(\mathrm{r}=.65^{* * *}\right)$, compromiso con sus convicciones $\left(\mathrm{r}=.48^{* * *}\right)$, confianza en sí mismo $\left(\mathrm{r}=.70^{* * *}\right)$, propensión al riesgo $\left(\mathrm{r}=.55^{* * *}\right)$, capacidad de adaptación $\left(\mathrm{r}=.58^{* * *}\right)$, Liderazgo $\left(\mathrm{r}=.66^{* * *}\right)$, independencia 
y autonomía $\left(\mathrm{r}=.38^{* * *}\right)$, necesidad de logro $(\mathrm{r}=.49 * * *)$, visión del futuro $(\mathrm{r}=.62 * * *)$, competencias $\left(\mathrm{r}=.69^{* * *}\right)$; es decir, a mayor optimismo mayor creatividad e iniciativa, perseverancia, compromiso con sus convicciones, confianza en sí mismo, mayor propensión al riesgo, mayor capacidad de adaptación, liderazgo, independencia y autonomía, necesidad de logro, visión del futuro y mayores competencias.

Tabla N. ${ }^{\circ}$ 4. Correlación entre compromiso con sus convicciones y los demás factores del emprendimiento.

\begin{tabular}{lcc}
\hline & \multicolumn{2}{c}{$\begin{array}{c}\text { COMPROMISO CON SUS } \\
\text { CONVICCIONES }\end{array}$} \\
\cline { 2 - 3 } & $\mathbf{r}$ & p-value \\
\hline Creatividad e iniciativa & 0.49014013 & 0.000001 \\
Optimismo & 0.48245231 & 0.000001 \\
Perseverancia & 0.52051532 & 0.000001 \\
Compromiso con sus convicciones & 1 & $\cdot$ \\
Confianza en sí mismo & 0.53174951 & 0.000001 \\
Propensión al riesgo & 0.52247209 & 0.000001 \\
Capacidad de adaptación & 0.59681857 & 0.000001 \\
Liderazgo & 0.48481844 & 0.000001 \\
Independencia y autonomía & 0.27100263 & 0.00021532 \\
Necesidad de logro & 0.35049567 & $1.2307 \mathrm{E}-06$ \\
Visión del futuro & 0.44362254 & 0.000001 \\
Competencias & 0.46279698 & 0.000001 \\
\hline
\end{tabular}

Tabla N. ${ }^{0}$ 5. Correlación entre optimismo y los demás factores del emprendimiento.

\begin{tabular}{lcc}
\hline & \multicolumn{2}{c}{ OPTIMISMO } \\
\cline { 2 - 3 } & $\mathbf{r}$ & $\mathbf{p}$-value \\
\hline Creatividad e iniciativa & 0.58231524 & 0.000001 \\
Optimismo & 1 & $\cdot$ \\
Perseverancia & 0.65497568 & 0.000001 \\
Compromiso con sus convicciones & 0.48245231 & 0.000001 \\
Confianza en sí mismo & 0.70328241 & 0.000001 \\
Propensión al riesgo & 0.55090841 & 0.000001 \\
Capacidad de adaptación & 0.58289789 & 0.000001 \\
Liderazgo & 0.65836607 & 0.000001 \\
Independencia y autonomía & 0.37804575 & 0.000001 \\
Necesidad de logro & 0.49055332 & 0.000001 \\
Visión del futuro & 0.61698708 & 0.000001 \\
Competencias & 0.68724023 & 0.000001 \\
\hline
\end{tabular}


La Tabla $\mathrm{N}^{\circ} 6$ nos muestra que existe una correlación significativa y positiva entre confianza en sí mismo y creatividad e iniciativa $\left(\mathrm{r}=.65^{* * *}\right)$, optimismo $\left(\mathrm{r}=.70^{* * *}\right)$ perseverancia $(\mathrm{r}=.74 * * *)$, compromiso con sus convicciones $(\mathrm{r}=.53 * * *)$, propensión al riesgo $(\mathrm{r}=.70 * * *)$, capacidad de adaptación $(\mathrm{r}=.69 * * *)$, liderazgo $(\mathrm{r}=.69 * * *)$, independencia y autonomía $\left(\mathrm{r}=.46^{* * *}\right)$, necesidad de logro $(\mathrm{r}=.44 * * *)$, visión del futuro $(\mathrm{r}=.69 * * *)$, competencias $\left(\mathrm{r}=.67^{* * *}\right)$; es decir, a mayor confianza en sí mismo mayor creatividad e iniciativa, optimismo, perseverancia, compromiso con sus convicciones, mayor propensión al riesgo, mayor capacidad de adaptación, liderazgo, independencia y autonomía, necesidad de logro, visión del futuro y mayores competencias.

Tabla $\mathbf{N}^{0}$ 6. Correlación entre la confianza en sí mismo y los demás factores del emprendimiento.

\begin{tabular}{lcc}
\hline & \multicolumn{2}{c}{ CONFIANZA EN Sí MISMO } \\
\cline { 2 - 3 } & $\mathbf{r}$ & p-value \\
\hline Creatividad e iniciativa & 0.64794111 & 0.000001 \\
Optimismo & 0.70328241 & 0.000001 \\
Perseverancia & 0.73731948 & 0.000001 \\
Compromiso con sus convicciones & 0.53174951 & 0.000001 \\
Confianza en sí mismo & 1 &. \\
Propensión al riesgo & 0.69716966 & 0.000001 \\
Capacidad de adaptación & 0.68700731 & 0.000001 \\
Liderazgo & 0.69146652 & 0.000001 \\
Independencia y autonomía & 0.45999071 & 0.000001 \\
Necesidad de logro & 0.43725687 & 0.000001 \\
Visión del futuro & 0.6918116 & 0.000001 \\
Competencias & 0.67363063 & 0.000001 \\
\hline
\end{tabular}

La Tabla N. ${ }^{o} 7$ nos muestra que existe una correlación significativa y positiva entre propensión al riesgo y creatividad e iniciativa $(\mathrm{r}=.57 * * *)$, optimismo $(\mathrm{r}=.55 * * *)$ perseverancia $\left(\mathrm{r}=.58^{* * *}\right)$, compromiso con sus convicciones $(\mathrm{r}=.52 * * *)$, confianza en sí mismo $(\mathrm{r}=.69 * * *)$, capacidad de adaptación $\left(\mathrm{r}=.66^{* * *}\right)$, liderazgo $(\mathrm{r}=.54 * * *)$, independencia y autonomía $(\mathrm{r}=.44 * * *)$, necesidad de logro $\left(\mathrm{r}=.48^{* * *}\right)$, visión del futuro $(\mathrm{r}=.62 * * *)$, competencias $(\mathrm{r}=.50 * * *)$; es decir, a mayor propensión al riesgo mayor creatividad e iniciativa, optimismo, perseverancia, compromiso con sus convicciones, mayor confianza en sí mismo, mayor capacidad de adaptación, liderazgo, independencia y autonomía, necesidad de logro, visión del futuro y mayores competencias. 
Tabla $\mathbf{N}^{0}$ 7. Correlación entre la propensión al riesgo y los demás factores del emprendimiento

\begin{tabular}{lcc}
\hline & PROPENSIÓN AL RIESGO & \\
\cline { 2 - 3 } & r & p-value \\
\hline Creatividad e iniciativa & 0.56800749 & 0.000001 \\
Optimismo & 0.55090841 & 0.000001 \\
Perseverancia & 0.58466438 & 0.000001 \\
Compromiso con sus convicciones & 0.52247209 & 0.000001 \\
Confianza en sí mismo & 0.69716966 & 0.000001 \\
Propensión al riesgo & 1 &. \\
Capacidad de adaptación & 0.65758868 & 0.000001 \\
Liderazgo & 0.53971374 & 0.000001 \\
Independencia y autonomía & 0.43554424 & 0.000001 \\
Necesidad de logro & 0.47558844 & 0.000001 \\
Visión del futuro & 0.62305741 & 0.000001 \\
Competencias & 0.50064754 & 0.000001 \\
\hline
\end{tabular}

La Tabla N. ${ }^{\circ} 8$ nos muestra que existe una correlación significativa y positiva entre capacidad de adaptación y creatividad e iniciativa $(\mathrm{r}=.54 * * *)$, optimismo $(\mathrm{r}=.58 * * *)$ perseverancia $\left(\mathrm{r}=.65^{* * *}\right)$, compromiso con sus convicciones $(\mathrm{r}=.60 * * *)$, confianza en sí mismo $\left(\mathrm{r}=.69^{* * *}\right)$, propensión al riesgo $\left(\mathrm{r}=.66^{* * *}\right)$, liderazgo $\left(\mathrm{r}=.58^{* * *}\right)$, independencia y autonomía $\left(\mathrm{r}=.36^{* * *}\right)$, necesidad de logro $\left(\mathrm{r}=.45^{* * *}\right)$, visión del futuro $(\mathrm{r}=.59 * * *)$, competencias $\left(\mathrm{r}=.56^{* * *}\right)$; es decir, a mayor capacidad de adaptación mayor creatividad e iniciativa, optimismo, perseverancia, compromiso con sus convicciones, mayor confianza en sí mismo, propensión al riesgo, liderazgo, independencia y autonomía, necesidad de logro, visión del futuro y mayores competencias.

Tabla N. ${ }^{\circ}$ 8. Correlación entre la capacidad de adaptación y los demás factores del emprendimiento.

\begin{tabular}{lcc}
\hline & CAPACIDAD DE ADAPTACIÓN \\
& r & p-value \\
\hline Creatividad e iniciativa & 0.53570237 & 0.000001 \\
Optimismo & 0.58289789 & 0.000001 \\
Perseverancia & 0.64833287 & 0.000001 \\
Compromiso con sus convicciones & 0.59681857 & 0.000001 \\
Confianza en sí mismo & 0.68700731 & 0.000001 \\
Propensión al riesgo & 0.65758868 & 0.000001 \\
Capacidad de adaptación & 1 &. \\
Liderazgo & 0.58084443 & 0.000001 \\
Independencia y autonomía & 0.36144871 & 0.000001 \\
Necesidad de logro & 0.45386298 & 0.000001 \\
Visión del futuro & 0.58759519 & 0.000001 \\
Competencias & 0.55662454 & 0.000001 \\
\hline
\end{tabular}


La Tabla $\mathrm{N}^{\circ} 9$ nos muestra que existe una correlación significativa y positiva entre el liderazgo y la creatividad e iniciativa $\left(\mathrm{r}=.65^{* * *}\right)$, optimismo $\left(\mathrm{r}=.66^{* * *}\right)$ perseverancia $(\mathrm{r}=.54 * * *)$, compromiso con sus convicciones $\left(\mathrm{r}=.48^{* * *}\right)$, confianza en sí mismo $(\mathrm{r}=.69 * * *)$, propensión al riesgo $(\mathrm{r}=.54 * * *)$, capacidad de adaptación $(\mathrm{r}=.58 * * *)$, independencia y autonomía $\left(\mathrm{r}=.36^{* * *}\right)$, necesidad de logro $(\mathrm{r}=.52 * * *)$, visión del futuro $\left(\mathrm{r}=.58^{* * *}\right)$, competencias $\left(\mathrm{r}=.66^{* * *}\right)$; es decir, a mayor liderazgo mayor creatividad e iniciativa, optimismo, perseverancia, compromiso con sus convicciones, mayor confianza en sí mismo, propensión al riesgo, capacidad de adaptación, independencia y autonomía, necesidad de logro, visión del futuro y mayores competencias.

Tabla N. ${ }^{\circ}$ 9. Correlación entre liderazgo y los demás factores del emprendimiento.

\begin{tabular}{lcc}
\hline & \multicolumn{2}{c}{ LIDERAZGO } \\
& r & p-value \\
\hline Creatividad e iniciativa & 0.64525514 & 0.000001 \\
Optimismo & 0.65836607 & 0.000001 \\
Perseverancia & 0.54295948 & 0.000001 \\
Compromiso con sus convicciones & 0.48481844 & 0.000001 \\
Confianza en sí mismo & 0.69146652 & 0.000001 \\
Propensión al riesgo & 0.53971374 & 0.000001 \\
Capacidad de adaptación & 0.58084443 & 0.000001 \\
Liderazgo & 1 &. \\
Independencia y autonomía & 0.36323036 & 0.000001 \\
Necesidad de logro & 0.52130321 & 0.000001 \\
Visión del futuro & 0.57635168 & 0.000001 \\
Competencias & 0.66413452 & 0.000001 \\
\hline
\end{tabular}

La Tabla N. ${ }^{\circ} 10$ nos muestra que existe una correlación significativa y positiva entre Independencia y autonomía y creatividad e iniciativa $(\mathrm{r}=.50 * * *)$, optimismo $\left(\mathrm{r}=.39^{* * *}\right)$ perseverancia $\left(\mathrm{r}=.38^{* * *}\right)$, compromiso con sus convicciones $(\mathrm{r}=.27 * * *)$, confianza en sí mismo $\left(\mathrm{r}=.46^{* * *}\right)$, propensión al riesgo $(\mathrm{r}=.44 * * *)$, capacidad de adaptación $\left(\mathrm{r}=.36^{* * *}\right)$, liderazgo $\left(\mathrm{r}=.36^{* * *}\right)$, necesidad de logro $(\mathrm{r}=.64 * * *)$, visión del futuro $(\mathrm{r}=.44 * * *)$, competencias $(\mathrm{r}=.49 * * *)$; es decir, a mayor independencia y autonomía mayor creatividad e iniciativa, optimismo, perseverancia, compromiso con sus convicciones, mayor confianza en sí mismo, propensión al riesgo, capacidad de adaptación, liderazgo, independencia y autonomía, necesidad de logro, visión del futuro y mayores competencias. 
Tabla N. ${ }^{\circ}$ 10. Correlación entre independencia y autonomía y los demás factores del emprendimiento.

\begin{tabular}{lcc}
\hline & \multicolumn{2}{c}{ INDEPENDENCIA Y AUTONOMÍA } \\
\cline { 2 - 3 } & $\mathbf{r}$ & p-value \\
\hline Creatividad e iniciativa & 0.50160374 & 0.000001 \\
Optimismo & 0.37804575 & 0.000001 \\
Perseverancia & 0.37948882 & 0.000001 \\
Compromiso con sus convicciones & 0.27100263 & 0.00021532 \\
Confianza en sí mismo & 0.45999071 & 0.000001 \\
Propensión al riesgo & 0.43554424 & 0.000001 \\
Capacidad de adaptación & 0.36144871 & 0.000001 \\
Liderazgo & 0.36323036 & 0.000001 \\
Independencia y autonomía & 1 &. \\
Necesidad de logro & 0.63554538 & 0.000001 \\
Visión del futuro & 0.44147476 & 0.000001 \\
Competencias & 0.4865612 & 0.000001 \\
\hline
\end{tabular}

La Tabla N. ${ }^{\circ} 11$ nos muestra que existe una correlación significativa y positiva entre necesidad de logro y creatividad e iniciativa $(\mathrm{r}=.62 * * *)$, optimismo $(\mathrm{r}=.49 * * *)$ perseverancia $\left(\mathrm{r}=.41^{* * *}\right)$, compromiso con sus convicciones $\left(\mathrm{r}=.35^{* * *}\right)$, confianza en sí mismo $(\mathrm{r}=.44 * * *)$, propensión al riesgo $(\mathrm{r}=.48 * * *)$, capacidad de adaptación $\left(\mathrm{r}=.45^{* * *}\right)$, liderazgo $(\mathrm{r}=.52 * * *)$, independencia y autonomía $(\mathrm{r}=.64 * * *)$, visión del futuro $(\mathrm{r}=.52 * * *)$, competencias $\left(\mathrm{r}=.51^{* * *}\right)$; es decir, a mayor necesidad de logro mayor creatividad e iniciativa, optimismo, perseverancia, compromiso con sus convicciones, mayor confianza en sí mismo, propensión al riesgo, capacidad de adaptación, liderazgo, independencia y autonomía, visión del futuro y mayor competencia.

Tabla N. ${ }^{0}$ 11. Correlación entre necesidad de logro y los demásfactores del emprendimiento.

\begin{tabular}{lcc}
\hline & \multicolumn{2}{c}{ NECESIDAD DE LOGRO } \\
\cline { 2 - 3 } & $\mathbf{r}$ & $\mathbf{p}$-value \\
\hline Creatividad e iniciativa & 0.61715082 & 0.000001 \\
Optimismo & 0.49055332 & 0.000001 \\
Perseverancia & 0.41359165 & 0.000001 \\
Compromiso con sus convicciones & 0.35049567 & $1.2307 \mathrm{E}-06$ \\
Confianza en sí mismo & 0.43725687 & 0.000001 \\
Propensión al riesgo & 0.47558844 & 0.000001 \\
Capacidad de adaptación & 0.45386298 & 0.000001 \\
Liderazgo & 0.52130321 & 0.000001 \\
Independencia y autonomía & 0.63554538 & 0.000001 \\
Necesidad de logro & 1 &. \\
Visión del futuro & 0.5299226 & 0.000001 \\
Competencias & 0.50810157 & 0.000001 \\
\hline
\end{tabular}


La Tabla N. ${ }^{\circ} 12$ nos muestra que existe una correlación significativa y positiva entre visión del futuro y creatividad e iniciativa $(\mathrm{r}=.67 * * *)$, optimismo $(\mathrm{r}=.62 * * *)$ perseverancia $\left(\mathrm{r}=.58^{* * *}\right)$, compromiso con sus convicciones $(\mathrm{r}=.44 * * *)$, confianza en sí mismo $(\mathrm{r}=.69 * * *)$, propensión al riesgo $(\mathrm{r}=.62 * * *)$, capacidad de adaptación $(\mathrm{r}=.59 * * *)$, liderazgo $\left(\mathrm{r}=.58^{* * *}\right)$, independencia y autonomía $(\mathrm{r}=.44 * * *)$, necesidad de logro $(\mathrm{r}=.53 * * *)$, competencias $(\mathrm{r}=.69 * * *)$; es decir, a mayor visión del futuro mayor creatividad e iniciativa, optimismo, perseverancia, compromiso con sus convicciones, mayor confianza en sí mismo, propensión al riesgo, capacidad de adaptación, liderazgo, independencia y autonomía, necesidad de logro y mayor competencia.

Tabla N. ${ }^{0}$ 12. Correlación entre visión del futuro y los demás factores del emprendimiento.

\begin{tabular}{lcc}
\hline & \multicolumn{2}{c}{ VISIÓN DEL FUTURO } \\
\cline { 2 - 3 } & $\mathbf{r}$ & p-value \\
\hline Creatividad e iniciativa & 0.6703369 & 0.000001 \\
Optimismo & 0.61698708 & 0.000001 \\
Perseverancia & 0.58151162 & 0.000001 \\
Compromiso con sus convicciones & 0.44362254 & 0.000001 \\
Confianza en sí mismo & 0.6918116 & 0.000001 \\
Propensión al riesgo & 0.62305741 & 0.000001 \\
Capacidad de adaptación & 0.58759519 & 0.000001 \\
Liderazgo & 0.57635168 & 0.000001 \\
Independencia y autonomía & 0.44147476 & 0.000001 \\
Necesidad de logro & 0.5299226 & 0.000001 \\
Visión del futuro & 1 &. \\
Competencias & 0.69136218 & 0.000001 \\
\hline
\end{tabular}

La Tabla N. ${ }^{\circ} 13$ nos muestra que existe una correlación significativa y positiva entre competencias y creatividad e iniciativa $(\mathrm{r}=.62 * * *)$, optimismo $(\mathrm{r}=.69 * * *)$, perseverancia $(\mathrm{r}=.59 * * *)$, compromiso con sus convicciones $\left(\mathrm{r}=.46^{* * *}\right)$, confianza en sí mismo $\left(\mathrm{r}=.67^{* * *}\right)$, propensión al riesgo $\left(\mathrm{r}=.50^{* * *}\right)$, capacidad de adaptación $\left(\mathrm{r}=.56^{* * *}\right)$, liderazgo $\left(\mathrm{r}=.66^{* * *}\right)$, independencia y autonomía $\left(\mathrm{r}=.49^{* * *}\right)$, necesidad de logro $(\mathrm{r}=.51 * * *)$, visión del futuro $(\mathrm{r}=.69 * * *)$; es decir, a mayor competencia mayor creatividad e iniciativa, optimismo, perseverancia, compromiso con sus convicciones, mayor confianza en sí mismo, propensión al riesgo, capacidad de adaptación, liderazgo, independencia y autonomía, necesidad de logro y visón del futuro. 
Tabla $\mathbf{N}^{0}$ 13. Correlación entre competencias y los demás factores del emprendimiento.

\begin{tabular}{lcc}
\hline & \multicolumn{2}{c}{ COMPETENCIAS } \\
\cline { 2 - 3 } & $\mathbf{r}$ & p-value \\
\hline Creatividad e iniciativa & 0.6225503 & 0.000001 \\
Optimismo & 0.68724023 & 0.000001 \\
Perseverancia & 0.59059288 & 0.000001 \\
Compromiso con sus convicciones & 0.46279698 & 0.000001 \\
Confianza en sí mismo & 0.67363063 & 0.000001 \\
Propensión al riesgo & 0.50064754 & 0.000001 \\
Capacidad de adaptación & 0.55662454 & 0.000001 \\
Liderazgo & 0.66413452 & 0.000001 \\
Independencia y autonomía & 0.4865612 & 0.000001 \\
Necesidad de logro & 0.50810157 & 0.000001 \\
Visión del futuro & 0.69136218 & 0.000001 \\
Competencias & 1 &. \\
\hline
\end{tabular}

\section{Creatividad y emprendimiento según variables sociodemográficas}

En seguida se describe el comportamiento de los sujetos, de acuerdo a la intervención de las variables demográficas, organizacionales y sociales que resultaron significativas.

\section{Creatividad y emprendimiento según sexo}

No existe diferencia significativa entre hombres y mujeres con respecto a creatividad e iniciativa, optimismo, perseverancia, compromiso con sus convicciones, confianza en sí mismo, propensión al riesgo, capacidad de adaptación, liderazgo, independencia y autonomía, necesidad de logro, visión del futuro y competencias. Dicho de otro modo, tanto los hombres como las mujeres le dan la misma importancia a cada uno de los constructos, el género no influye en el concepto que manejan sobre los componentes.

\section{Creatividad y emprendimiento según grupos de edad}

Existe suficiente evidencia estadística para rechazar la hipótesis planteada a un nivel de significación de 0.05; por tanto, se concluye que existe diferencias entre los grupos de edad con respecto a lo que perciben u opinan sobre la propensión al riesgo (p-value $=.019$ ), visión del futuro ( $\mathrm{p}$-value $=.014)$ y competencias $(\mathrm{p}$-value $=.012)$; es decir, las personas agrupadas en rangos de edad mayores le dan más importancia (o peso) a los riesgos, al futuro y a las competencias. De la misma manera, pero a un nivel de significación de 0.10, 
resulta la diferencia que existe entre los grupos de edad respecto a lo que opinan sobre la confianza en sí mismo ( $p$-value $=.060)$, o sea, las personas agrupadas en rangos de edad mayores son los que le dan más peso a la confianza en sí mismo. Probablemente, debido a que estos componentes exigen mayor madurez para valorarlos en su real dimensión.

\section{Creatividad y emprendimiento según estado civil}

No existe diferencia significativa de acuerdo al estado civil de los estudiantes con respecto a creatividad e iniciativa, optimismo, perseverancia, compromiso con sus convicciones, confianza en sí mismo, propensión al riesgo, capacidad de adaptación, liderazgo, independencia y autonomía, necesidad de logro, visión del futuro y competencias. Dicho de otro modo, tanto los solteros, casados, como los de unión libre le dan la misma importancia a cada uno de los constructos; el estado civil no influye en el concepto que manejan sobre los componentes del emprendimiento.

\section{Creatividad y emprendimiento según el ciclo de estudios}

Existe suficiente evidencia estadística para rechazar la hipótesis planteada a un nivel de significación de 0.05; por tanto, se puede decir que existen diferencias entre los ciclos de estudio con respecto a lo que perciben u opinan sobre la perseverancia ( $\mathrm{p}$-value $=.048$ ) y competencias $(\mathrm{p}$-value $=.011)$. Es decir, las personas agrupadas en rangos por ciclo de estudios que van de VI a XII son las que le dan más importancia (o peso) a la perseverancia y a las competencias. De la misma manera, pero a un nivel de significación de 0.10 , existe diferencia entre rangos por ciclo de estudios respecto a lo que opinan sobre el optimismo $(\mathrm{p}$-value $=.095)$, o sea, las personas agrupadas en rangos por ciclo de estudios (VI a XIII) son las que le dan más peso al optimismo. Probablemente, porque dichos componentes son más próximos a los ciclos avanzados en la medida en que los estudiantes están por concluir sus estudios y encarar la realidad del ejercicio profesional.

\section{Creatividad y emprendimiento según la facultad de pertenencia}

Existe suficiente evidencia estadística para rechazar la hipótesis planteada a un nivel de significación de 0.10; por tanto, se concluye que existen diferencias entre los estudiantes de acuerdo a la facultad a la que pertenecen con respecto a lo que piensan sobre la perseverancia ( $\mathrm{p}$-value $=.076)$ y liderazgo $(\mathrm{p}$-value $=.061)$; es decir, los estudiantes agrupados en rangos por facultades de pertenencia son los que le dan más importancia a la perseverancia (psicología, química e ingeniería química y biología) y al liderazgo (psicología, minas y química e ingeniería química). Es posible que estos resultados se deban a que dichos estudiantes perciben que su Facultad está mejor posicionada en el mercado académico y/o laboral. 


\section{Creatividad y emprendimiento según la EAP o carrera profesional}

Existe suficiente evidencia estadística para rechazar la hipótesis planteada a un nivel de significación de 0.05; por tanto, se concluye que existen diferencias entre los estudiantes de acuerdo a la carrera profesional o EAP (Escuela Académico Profesional) a la que pertenecen con respecto a lo que piensan sobre el optimismo ( $\mathrm{p}$-value $=.043)$ y la perseverancia $(\mathrm{p}$-value $=.036)$; es decir, los estudiantes agrupados en carreras profesionales o EAP de pertenencia son los que le dan más importancia al optimismo (metalúrgica, psicología y genética y biotecnología) y a la perseverancia (psicología, química y metalúrgica). Y a un nivel de significación de 0.10 , los estudiantes de las carreras de metalúrgica, psicología, genética y biotecnología son los que le dan más peso a las competencias (p-value $=$ .057) del emprendimiento. Probablemente esté asociado a la percepción que tienen los estudiantes sobre su formación profesional, el plan de estudios de su carrera y su cercanía a las exigencias reales del mercado.

\section{Creatividad y emprendimiento según la experiencia laboral}

Existe suficiente evidencia estadística para rechazar la hipótesis planteada a un nivel de significación de 0.10; por tanto, se concluye que existen diferencias entre los estudiantes agrupados de acuerdo a su experiencia laboral con respecto a lo que piensan sobre la perseverancia $(\mathrm{p}$-value $=.076)$ y el liderazgo $(\mathrm{p}$-value $=.061)$; es decir, los estudiantes agrupados de acuerdo a su experiencia laboral son los que le dan más importancia a la perseverancia (independiente, dependiente y otras formas) y el liderazgo (independiente, sin experiencia y otras formas). Se debe, tal vez a la posibilidad que tienen los estudiantes de realizar actividades para probar sus habilidades profesionales con más libertad, lo que demanda de perseverancia y liderazgo.

\section{Creatividad y emprendimiento según piense desarrollar un emprendimiento}

La evidencia estadística nos permite rechazar la hipótesis planteada a un nivel de 0.05 ; por tanto, se puede decir que existe diferencia significativa entre los que piensan desarrollar un emprendimiento con respecto a creatividad e iniciativa ( $\mathrm{p}$-value $=.021$ ), optimismo $(\mathrm{p}$-value $=.002)$, perseverancia $(\mathrm{p}$-value $=.004)$, confianza en sí mismo $(\mathrm{p}$-value $=.003)$, capacidad de adaptación $(\mathrm{p}$-value $=.005)$, necesidad de logro $(\mathrm{p}$-value $=.006)$, visión del futuro ( $\mathrm{p}$-value $=.001)$ y competencias ( $\mathrm{p}$-value $=.010) ; \mathrm{y}$, a un nivel de 0.10, propensión al riesgo $(\mathrm{p}$-value $=.080)$, y el liderazgo $(\mathrm{p}$-value $=.120)$ que está cercano al nivel de significación 0.10 que puede afianzarse por tamaño de muestra u otros cambios de detalle.

En conclusión, estos resultados nos sugieren que aquellos sujetos que piensan desarrollar un emprendimiento han obtenido mayor peso en cada uno de los componentes o constructos de la creatividad y el emprendimiento. Esto reafirma la actitud positiva de los estudiantes hacia el emprendimiento. 
Creatividad y emprendimiento según el sector de actividad donde piensa desarrollar su emprendimiento

La evidencia estadística nos permite rechazar la hipótesis planteada a un nivel de 0.05 ; por tanto, se puede decir que existe diferencia significativa entre los rangos agrupados según el sector de actividad donde piensan desarrollar un emprendimiento con respecto a optimismo $(p$-value $=.007)$, perseverancia $(p$-value $=.045)$, liderazgo $(p$-value $=.026)$, y competencias ( $\mathrm{p}$-value $=.003$ ); por tanto, los sujetos que se agrupan por sectores de actividades para emprender son los que le dan más importancia a los factores de optimismo (salud, comercio y educación), perseverancia (salud, comercio y servicios), liderazgo (salud, comercio y educación), competencias (comercio, salud y educación). Probablemente, porque son actividades vinculadas directamente a las necesidades prioritarias de la población y por la probabilidad de ingresar al mercado con mayor éxito, si se tiene en cuenta dichos factores o componentes.

\section{DISCUSIÓN}

A la luz de la teoría y las investigaciones revisadas, si bien los componentes de la creatividad y el empredimiento permiten determinar las actitudes emprendedoras de las personas, los factores condicionantes tienen que ver, sin duda, con las condiciones socioculturales que ofrece el ambiente a los miembros de un prupo social o una sociedad, tales como la familia, la escuela, la universidad, el barrio y las experiencias laborales de la familia y las experiencias personales (F. A. Delicio, 2007), a los que se añaden las cualidades personales para la formación de actitudes y comportamientos emprendedores.

Este estudio nos indica, en términos generales, que los jóvenes estudiantes de la Universidad Nacional Mayor de San Marcos tienen una actitud positiva hacia la creatividad y el emprendedorismo, en la medida en que sus componentes son confiables y valorados por tales sujetos. Es natural que tratándose de jóvenes en proceso de formación no exista comportamiento emprendedor visible y que sus aspiraciones en la práctica se desvanezcan, porque su prioridad es concluir sus estudios, realizar sus prácticas profesionales $(\mathrm{N}$. Espinoza, 2004) y no la de abrir su propio negocio a corto plazo.

Por otro lado, Fernando Graña y Mariel Fornoni (2002) distinguen la vocación emprendedora e intención emprendedora de la actitud emprendedora, cuando por definición, todo ello no es sino una actitud (M. Olivero, 2007). De manera que se puede inferir que la actitud emprendedora es una tendencia positiva y alta, mientras que el comportamiento es acción, tiene que ver con realizaciones concretas (IEEM de la Universidad de Montevideo, 2007).

Las variables demográficas, organizacionales y sociales, por su lado, nos indican que la edad juega un papel importante en la valoración de los factores del emprendimiento, al asociarse con la confianza en sí mismo, la propensión al riesgo, la visión al futuro y las competencias, siendo más importante en quienes tienen más edad. Igualmente, los ciclos de estudio, la Facultad a la que pertenecen y la Escuela Académico Profesional donde siguen la carrera están asociadas a optimismo, perseverancia, liderazgo y competencias, 
demostrando el mayor peso que se le otorga a dichos componentes de acuerdo a la cercanía con la culminación de los estudios, la posición de la Facultad en el mercado académico y tal vez los planes de estudio acordes con la necesidad social y la realidad laboral. Y, finalmente, la experiencia laboral que se encuentra relacionada a perseverancia y liderazgo, con un peso mayor y un concepto diferente por aquellos que realizan actividad independiente; entre tanto, los que piensan desarrollar un emprendimiento están asociados a todos los componentes de la creatividad y el emprendimiento con un peso mayor y un concepto diferente, en relación con los que no piensan realizar actividades de emprendimiento. Aquellos que piensan realizar su emprendimiento, incursionarían especialmente en actividades del sector comercio y salud, quienes le otorgan mayor peso al optimismo, la perseverancia, el liderazgo y las competencias, probablemente, porque son actividades vinculadas directamente a las necesidades prioritarias de la población y por la probabilidad de ingresar al mercado con mayor éxito, si se tienen en cuenta dichos factores o componentes.

Estos resultados nos demuestran la capacidad predictiva del instrumento; sin embargo, sólo la administración a una muestra más amplia en diferentes ámbitos de la actividad académica con jóvenes estudiantes puede confirmar la validez del instrumento. Es posible también que los resultados varíen en algunos aspectos con la ampliación de la muestra; noobstante, es pertinente hacer algunos ajustes a las variables demográficas para obtener y aprovechar mejor los resultados; existen vacíos que deben ser explorados añadiendo variables relacionadas, por ejemplo, a la procedencia del estudiante y a la de su familia y otras variables que, sin duda, serán de mucha utilidad para ir consolidando estos resultados.

\section{CONCLUSIONES}

1. Los estudiantes de la Universidad Nacional Mayor de San Marcos tienen una actitud positiva en un nivel alto con tendencia a incrementarse al nivel muy alto.

2. Una exploración de cada uno de los componentes de la creatividad y el emprendimiento nos indica que existe confiabilidad de cada uno de los componentes, haciendo consistente la correlación positiva y significativa entre ellos.

3. Existe asociación de algunos componentes de la creatividad y el emprendimiento con variables demográficas, tales como:

- La propensión al riesgo, la visión del futuro, las competencias personales y la confianza en sí mismo están asociadas a la edad, siendo las personas agrupadas en rangos de edad mayores los que le dan más importancia (o peso).

- La perseverancia, competencias personales y optimismo están relacionadas a los ciclos de estudio; siendo los sujetos agrupados en rangos por ciclo de estudios que van de VI a XII los que le dan más importancia (o peso) a dichos componentes.

- La perseverancia y el liderazgo están asociados a la Facultad a la que pertenecen; es decir, los estudiantes agrupados en rangos por Facultades de pertenencia tales como 
psicología, química e ingeniería química y biología son los que le dan más importancia a la perseverancia; y psicología, minas y química e ingeniería química los que le dan más peso al liderazgo.

- El optimismo y la perseverancia están asociados a las Escuelas Académico Profesionales (EAP) a la que pertenecen; siendo los estudiantes agrupados en las EAP de metalúrgica, psicología y genética y biotecnología los que le dan más importancia al optimismo y aquellos de psicología, química y metalúrgica a la perseverancia. Y son los que pertenecen a las EAP de metalúrgica, psicología y genética y biotecnología los que le dan más peso a las competencias personales.

- La perseverancia y el liderazgo están relacionados con la experiencia laboral; es decir, los estudiantes agrupados de acuerdo a su experiencia laboral son los que le dan más importancia a la perseverancia (actividades independientes, dependientes y otras formas) y el liderazgo (actividades independientes, sin experiencia y otras formas).

- La creatividad e iniciativa, el optimismo, la perseverancia, la confianza en sí mismo, la capacidad de adaptación, la necesidad de logro, la visión del futuro, las competencias, y la propensión al riesgo, están asociados a quienes piensan desarrollar un emprendimiento, excepto el liderazgo que está cercano al nivel de significación y que puede afianzarse dependiendo de la muestra u otros cambios. Esta situación demuestra que los estudiantes dispuestos a desarrollar un emprendimiento le dan mayor importancia a dichos componentes del emprendimiento, reafirmando la actitud positiva de los estudiantes hacia la creatividad y el emprendimiento.

- Finalmente, el sector de actividad donde pueden desarrollar su emprendimiento está asociado a optimismo, perseverancia, liderazgo y competencias; por tanto, los sujetos que se agrupan por sectores de actividades para emprender son los que le dan más importancia a dichos factores: optimismo (salud, comercio y educación), perseverancia (salud, comercio y servicios), liderazgo (salud, comercio y educación), competencias (comercio, salud y educación).

\section{REFERENCIAS BIBLIOGRÁFICAS}

1. Bóveda, Juan (2004). El emprendedor y las pequeñas empresas. Fundación EMPREDER. Rev. Futuros, Vol II(6). Paraguay: http://www.revistafuturos.inf

2. Cevallos V. Emilio (2007). Restricciones del entorno a la competitividad empresarial en América Latina. Cochabamba, Bolivia: FUNDES Internacional

3. Centro de Desarrollo Emprendedor del Instituto de Estudios Empresariales de Montevideo (IEEM) (2007). Emprendedorismo en los universitarios: Una opción cada día más real. Montevideo. Uruguay: Universidad de Montevideo.

4. Delicio, F. (2007). La vocación emprendedora de los estudiantes universitarios: El Caso de los estudiantes de la Facultad de Ciencias Económicas y Sociales de la Universidad Nacional de Mar del Plata. Argentina: UNMDP, Mar del Plata, 
5. Espinoza, N. (2004). Las aptitudes y actitudes empresariales de los estudiantes de administración en las universidades publicas de Lima y Callao. Perú: Facultad de Ciencias Administrativas, UNMSM.

6. Graña, F. y Fornoni, M. (2002). La vocación emprendedora en los alumnos universitarios avanzados de Ciencias Económicas de la ciudad de Mar del Plata. Argentina: UNMDP, Mar del Plata.

7. Moriano. J. (2005). El perfil psicosocial del emprendedor. Rev. Ministerio de Trabajo y Asuntos Sociales. Madrid, España: CES Colección Estudios.

8. Shiersmann, S.; Graña, F. y Liseras, N. (2002). Vocación emprendedora en alumnos universitarios avanzados: El caso de las Facultades de Ciencias Económicas de FASTA y de la UNMDP. VII Reunión Anual Red Pymes Mercosur, Rafaela. Publicación en cd-rom. 